\title{
Effectiveness and Economic Outcomes in Patients Undergoing Laparoscopic Prostate Resection with a New Surgical Shear with Integrated Energy System: a retrospective study based on a tertiary hospital database in China
}

\section{Yi Gao}

Ruijin Hospital

Yu Zhu

Ruijin Hospital

\section{Fukang Sun}

Ruijin Hospital

\section{Yuan Shao}

Ruijin Hospital

\section{Tao Huang}

Ruijin Hospital

Wei He

Ruijin Hospital

Xin Xie

Ruijin Hospital

Lu Chen

Ruijin Hospital

Debra R. Winberg

Tulane School of Public Health and Tropical Medicine, Department of Health Policy and Management Danfeng Xu ( $\nabla$ xdf12036@rjh.com.cn)

Ruijin Hospital

\section{Research Article}

Keywords: laparoscopic prostate resection, surgical shears, Harmonic ACE +7 , clinical effectiveness, costeffectiveness

Posted Date: August 4th, 2022

DOl: https://doi.org/10.21203/rs.3.rs-1361268/v2 
License: (c) (i) This work is licensed under a Creative Commons Attribution 4.0 International License. Read Full License 


\section{Abstract \\ Background}

Each year over 1.4 million men are diagnosed with prostate cancer. Surgical prostate resection offers patients with early-stage prostate cancer a high likelihood of remission and has low reoperation rates. A new surgical shear with integrated energy system (Harmonic ACE +7 ) is an integrated ultrasonic and electrosurgical energy system that can aid surgeons in laparoscopic prostate resections. This study aimed to demonstrate the ACE + 7's value by determining its effectiveness and economic outcomes compared to conventional ultrasonic shear (CUS) in a real-world setting.

\section{Methods}

This was a multi-center clinical study of adults with prostate cancer undergoing a laparoscopic prostate resection procedure with the ACE + 7 shear or CUS between August 2019 and April 2021 at Shanghai Ruijin Hospital and two affiliated centers. Demographic and diagnosis information, intraoperative and postoperative clinical outcomes, and total and categorical costs were collected. 1:1 Propensity Score Matching was performed to form the study population for each clinical group. Data were compared between the two groups using t-tests and chi-squared tests.

\section{Results}

The ACE + 7 was associated with a lower number of hemostatic clips used per surgery (12.8 in the ACE + 7 group vs 19.8 in the CUS group, $P<0.001)$, a moderate but not significant difference in average postoperative drainage duration $(6.6 \pm 2.2 \mathrm{~d}$ vs. $7.9 \pm 4.1 \mathrm{~d}, \mathrm{P}=0.082)$, a reduction on total drainage volume $(275.5 \pm 374.3 \mathrm{ml}$ vs. $492.9 \pm 1495.0 \mathrm{ml}, \mathrm{P}=0.321)$ and a lower average rate of postoperative hemostatic drug usage ( $16 \%$ vs. $52 \%, \mathrm{P}<0.001)$. There was no significant difference in total costs between the ACE +7 and CUS groups.

\section{Conclusion}

This study provides real-world data demonstrating that the ACE + 7 shear with integrated energy system improves clinical outcomes compared to CUS and can offer cost savings for hospitals and health systems. Using the ACE + 7's during LPRs allows physicians to help their patients achieve better outcomes and not spend additional money.

\section{Trial Registration:}


This study is a retrospective, observational study based on data from existing Electronical Hospital Information System and no human intervention was involved.

\section{Background}

Each year over 1.4 million men are diagnosed with prostate cancer [1]. Prostate cancer is the second most common cancer in men and has a higher burden in men over 65 . Therefore, its incidence is expected to rise as life expectancy increases [2]. Although improved therapies and diagnostic tools have decreased prostate-cancer related mortality, access to innovative, lifesaving prostate cancer care is not available worldwide [2, 3]. In turn, prostate cancer's incidence and mortality rates are rising in some countries such as China [4]. As the burden of prostate cancer grows in the coming years, it is essential to ensure people have access to clinically effective and cost-effective treatments regardless of where they live. Through effective care, people worldwide can live long and productive lives.

Treatments for prostate cancer include surgery, radiation therapy, high-intensity focused ultrasound, cryotherapy, chemotherapy, and immunotherapy [2]. Physicians choose a treatment to use based on the patient's disease progress [2]. For example, in China, surgical prostate resections are recommended for the management of resectable prostate cancer [5]. Surgical prostate resection offers patients with earlystage prostate cancer a high likelihood of remission and has low reoperation rates. Most Chinese surgeons perform this surgery laparoscopically as laparoscopic prostate resections (LPRs) are less invasive than open prostatectomies, decrease operation time, decrease blood loss, have a lower risk of causing postoperative urinary leakages, and have a lower risk of causing sexual dysfunction [6]. However, parts of the procedure, including the resection of collateral ligaments, are very technically demanding for surgeons [7]. In response to these difficulties, researchers are constantly developing new surgical techniques and tools to make LPRs safer.

One example of these tools is a new surgical shear with integrated energy system (Harmonic ACE +7$)$. It is an integrated ultrasonic and electrosurgical energy system that leverages adaptive tissue technology with predictive analytics to modulate energy delivery during the sealing cycle [6]. With greater burst pressures than those from older bipolar technologies, the Harmonic ACE + 7 shear with advanced hemostasis mode can seal vessels up to $7 \mathrm{~mm}$ in diameters [7]. Several clinical trials demonstrate the $\mathrm{ACE}+7$ 's hemostasis efficacy, ability to seal large vessels, and its lower thermal energy output [6-9]. Furthermore, the innovative device can improve operative efficiency by eliminating the need for instrument exchanges during surgery, making it best suited for surgeries that require dissection, mobilization, and large vessel sealing [10]. Thus, it could be a useful tool for LPRs.

Although current evidence establishes the ACE + 7's clinical benefits, few studies have used real-world data to assess the economic value of the $A C E+7$. For more surgeons to use the $A C E+7$, it is essential to offset the premium price by showing the $A C E+7$ is a high-value, cost-effective surgical tool. This study aimed to demonstrate the ACE +7 's's value by determining its effectiveness and economic outcomes compared to conventional ultrasonic shears (CUS) in LPRs in a real-world setting. 


\section{Methods}

\section{Study Population and Data Source}

This is a multi-center observational study of adults ( $\geq 18$ years old) with prostate cancer undergoing an LPR procedure with the ACE+7 shear or CUS between August 2019 and April 2021 at Shanghai Ruijin Hospital, including the headquarters, the North Center and Luwan Center in China. Patients were excluded from the study if they had a robotic-assisted surgery or an intra-fascial prostate resection.

The demographic, diagnosis, and surgical information, intraoperative and postoperative clinical outcomes, and hospitalization costs were collected retrospectively through an Electronic Hospital Information System (HIS). Data extraction was performed by trained physicians and validated by a research assistant to ensure the data's accuracy. The study was reviewed and approved by Ethical Committee in September 2021.

Surgeon preference dictated which type of ultrasonic shear was used during surgery. 1:1 Propensity Score Matching (PSM) was performed to form the study population for each clinical group (ACE+7 or CUS). PSM is a standard, widely applied technique that simulates an experimental study to estimate a causal effect in an observational data set [11]. It attempts to control confounding biases by making the groups receiving treatment and no treatment comparable to the control variables. Propensity scoring was performed using age, study site, BMI, comorbidities, abnormal rate of coagulation, Gleason score, lymph node dissection, and pelvic adhesiolysis.

\section{Clinical Effectiveness}

Researchers assessed several endpoints to evaluate the clinical effectiveness of the ACE +7 shear with integrated energy system compared to the CUS. These included intraoperative hemostatic clip usage, intraoperative blood loss, transfusion rate, operation time, postoperative rate of using hemostatic drugs, postoperative length of drainage, postoperative drainage volume, length of stay (LOS), postoperative LOS, re-operation rate during index hospitalization, and postoperative readmission within 30 days.

\section{Hospitalization Costs}

To determine the cost-effectiveness of the ACE +7 compared to CUS, direct medical costs during index hospitalization on each patient was collected through an HIS. Direct hospitalization costs included total cost and the cost breakdown comprised of device cost, treatment cost, pharmaceutical cost, nursing cost, board and room cost, lab test cost, examination cost, transfusion cost, oxygen therapy cost, traditional Chinese medicine (TCM) cost, diagnosis cost, and other costs.

\section{Statistical Analysis}

Descriptive analysis of the baseline characteristics was performed before and after PSM. Mean and standard deviation were tabulated for continuous variables. Frequency and percentage were tabulated for 
categorical variables. Statistical differences of continuous variables between the ACE +7 and CUS groups were analyzed by a t-test. Categorical outcome variables were compared using a Chi-square test when the expected frequency is greater than 5 for all cells or a Fisher exact test when the expected frequency is less than 5 in at least one of the cells. Hospitalization costs were compared between the two groups using a t-test.

All statistical analyses were performed using $\mathrm{R}$ software version 3.5.3, with $\mathrm{P}$ values $<0.05$ considered as statistically significant.

\section{Results}

A total of 222 cases met the inclusion and exclusion criteria. After excluding patients with missing data, 193 patients were included for descriptive analysis at baseline, with 70 in the ACE +7 group and 123 in the CUS group (Fig. 1).

Baseline characteristics were compared in the initial 193 patients. It was found that the distribution of study site was statistically different between the two groups. The percent of preoperative abnormal coagulation function and the percent of lymph node dissection during surgery were statistically higher in ACE +7 group compared to CUS group (Table 1).

Table 1

Baseline characteristics comparisons between $\mathrm{ACE}+7$ group and CUS group

\begin{tabular}{|llll|}
\hline Baseline characteristics & $\begin{array}{l}\text { ACE + 7 group (N= } \\
\text { Age, mean } \pm \text { SD }\end{array}$ & $\begin{array}{l}\text { CUS group (N= } \\
\text { 123) }\end{array}$ & $\begin{array}{l}\mathbf{P} \\
\text { value }\end{array}$ \\
\hline Study site, N(\%) & $68.6 \pm 6.6$ & $68.9 \pm 6.5$ & 0.736 \\
\hline Ruijin Hospital and North Center & $35(50.0 \%)$ & $88(71.5 \%)$ & 0.005 \\
\hline Ruijin Luwan center & $35(50.0 \%)$ & $35(28.5 \%)$ & \\
\hline BMI(kg/m²), mean \pm SD & $24.5 \pm 2.4$ & $23.9 \pm 2.7$ & 0.156 \\
\hline Comorbidity of hypertension, N(\%) & $34(48.6 \%)$ & $61(49.6 \%)$ & 1.000 \\
\hline Comorbidity of diabetes, N(\%) & $13(18.6 \%)$ & $18(14.6 \%)$ & 0.608 \\
\hline $\begin{array}{l}\text { Pre-op abnormal coagulation function, } \\
\text { N(\%) }\end{array}$ & $36(51.4 \%)$ & $42(34.1 \%)$ & 0.028 \\
\hline $\begin{array}{l}\text { Gleason score, mean } \pm \text { SD } \\
\text { Lymph node dissection during surgery, }\end{array}$ & $57(81.4 \%)$ & $7.3 \pm 0.9$ & 0.115 \\
\hline N(\%) & $35(50.0 \%)$ & $63(51.2 \%)$ & $<0.001$ \\
\hline Pelvic adhesiolysis during surgery, N(\%) & $58(47.2 \%)$ & 0.818 \\
\hline
\end{tabular}


After 1:1 PSM, there were 50 patients in each treatment group with no statistical difference in baseline characteristics between the groups (Table 2). The average age for patients in the ACE +7 group was 68.2 \pm 6.2 years, and $68.4 \pm 7.1$ years for the CUS group. Rates of underlying conditions were similar between groups. In the ACE +7 group, 26 (52\%) patients had hypertension, and 9 (40\%) were diabetic. In comparison, 25 (50\%) of the CUS group had hypertension, and $21(42 \%)$ were diabetic. Patients in each group had similar disease progression with 7.6 vs. 7.7 Gleason scores in the ACE + 7 and CUS groups, respectively.

Table 2

Baseline characteristics comparisons between ACE +7 group and CUS group after 1:1 Propensity Score Matching

\begin{tabular}{|llll|}
\hline Baseline characteristics & $\begin{array}{l}\text { ACE + 7 group (N= } \\
\text { 50) }\end{array}$ & $\begin{array}{l}\text { CUS group (N= } \\
\mathbf{5 0})\end{array}$ & P value \\
\hline Age, mean \pm SD & $68.2 \pm 6.2$ & $68.4 \pm 7.1$ & 0.858 \\
\hline Study site, N(\%) & & & 0.682 \\
\hline Ruijin Hospital and North Center & $32(64.0 \%)$ & $29(58.0 \%)$ & \\
\hline Ruijin Luwan center & $18(36.0 \%)$ & $21(42.0 \%)$ & \\
\hline BMI(kg/m²), mean \pm SD & $24.3 \pm 2.6$ & $23.6 \pm 3.2$ & 0.224 \\
\hline Comorbidity of hypertension, N(\%) & $26(52.0 \%)$ & $25(50.0 \%)$ & 1.000 \\
\hline Comorbidity of diabetes, N(\%) & $9(18.0 \%)$ & $7(14.0 \%)$ & 0.785 \\
\hline $\begin{array}{l}\text { Pre-op abnormal coagulation function, } \\
\text { N(\%) }\end{array}$ & $20(40.0 \%)$ & $21(42.0 \%)$ & 1.000 \\
\hline Gleason score, mean \pm SD & $7.6 \pm 1.0$ & $7.7 \pm 1.0$ & 0.477 \\
\hline $\begin{array}{l}\text { Lymph node dissection during surgery, } \\
\text { N(\%) }\end{array}$ & $39(78.0 \%)$ & $37(74.0 \%)$ & 0.815 \\
\hline Pelvic adhesiolysis during surgery, N(\%) & $32(64.0 \%)$ & $25(50.0 \%)$ & 0.226 \\
\hline
\end{tabular}

\section{Clinical Effectiveness}

The number of hemostatic clips used per surgery was significantly different between the ACE +7 and the CUS groups. Surgeons, on average, used 12.8 hemostatic clips for patients in the ACE +7 group compared to 19.8 clips for patients in the CUS group $(P<0.001)$. There was a moderate but not significant difference in average postoperative drainage duration: $6.6 \pm 2.2 \mathrm{~d}$ in the ACE +7 group vs. 7.9 $\pm 4.1 \mathrm{~d}$ in the CUS group $(P=0.082)$. Additionally, there was nearly a $50 \%$ reduction in average drainage volume in the ACE + 7 group compared with the CUS group: total drainage volume: $275.5 \pm 374.3 \mathrm{ml}$ in the ACE +7 group vs. $492.9 \pm 1495.0 \mathrm{ml}$ in the CUS group, $(P=0.321)$. The $A C E+7$ group had a significantly lower average postoperative hemostatic drug usage rate: $16 \%$ vs. $52 \%(P<0.001)$. No significant 
differences were found in the amount of blood loss, the transfusion rate, the operation time, or the LOS (Table 3).

\section{Table 3}

Clinical Outcomes Comparisons between ACE +7 group and CUS after PSM

\begin{tabular}{|c|c|c|c|}
\hline Endpoints & $\begin{array}{l}\text { ACE }+7 \\
\text { group } \\
(N=50)\end{array}$ & $\begin{array}{l}\text { CUS group } \\
(\mathrm{N}=50)\end{array}$ & $\begin{array}{l}P \\
\text { value }\end{array}$ \\
\hline Intraoperative hemostatic clip usage, mean \pm SD & $12.8 \pm 5.8$ & $19.8 \pm 6.6$ & $<0.001$ \\
\hline Intraoperative blood loss $(\mathrm{mL})$, mean \pm SD & $191.1 \pm 152.8$ & $204.2 \pm 181.6$ & 0.782 \\
\hline Transfusion rate, $\mathrm{N}(\%)$ & $1(2.0 \%)$ & $3(6.0 \%)$ & 0.610 \\
\hline Operation time $(\min )$, mean \pm SD & $162.6 \pm 44.8$ & $158.0 \pm 37.4$ & 0.586 \\
\hline Post-op rate of hemostatic drug usage, $\mathrm{N}(\%)$ & $8(16.0 \%)$ & $26(52.0 \%)$ & $<0.001$ \\
\hline Post-op length of drainage (days), mean \pm SD & $6.6 \pm 2.2$ & $7.9 \pm 4.1$ & 0.082 \\
\hline $\begin{array}{l}\text { Post-op drainage volume within } 24 \text { hours }(\mathrm{mL}) \text {, mean } \pm \\
\text { SD }\end{array}$ & $72.0 \pm 59.1$ & $133.4 \pm 282.0$ & 0.135 \\
\hline $\begin{array}{l}\text { Post-op drainage volume within } 48 \text { hours }(\mathrm{mL}) \text {, mean } \pm \\
\text { SD }\end{array}$ & $143.4 \pm 135.5$ & $239.6 \pm 495.9$ & 0.189 \\
\hline Post-op total drainage volume $(\mathrm{mL})$, mean \pm SD & $275.5 \pm 374.3$ & $\begin{array}{l}492.9 \pm \\
1495.0\end{array}$ & 0.321 \\
\hline Length of stay (days), mean \pm SD & $10.7 \pm 3.4$ & $11.3 \pm 4.8$ & 0.413 \\
\hline Postoperative length of stay (days), mean \pm SD & $7.9 \pm 3.0$ & $8.6 \pm 4.2$ & 0.337 \\
\hline Re-operation during index hospitalization, $\mathrm{N}(\%)$ & 0 & 0 & NA \\
\hline Post-op readmission within 30 days, $\mathrm{N}(\%)$ & 0 & 0 & NA \\
\hline
\end{tabular}

\section{Hospitalization Costs}

Although the price of the ACE +7 shear is higher than that of the CUS, there was no significant difference in total cost between the ACE +7 group and the CUS group: RMB 42,675 vs. RMB 41,426 ( $P=0.349)$ (Table 4). This was explained by cost savings in several line items, including treatment costs, pharmaceutical costs, lab test costs, exam costs, board and room costs, nursing costs, transfusion costs, and oxygen therapy costs (Table 4). The total cost excluding intervention cost was RMB 38357.8 in the ACE + 7 group compared to RMB 39983.1 in the CUS group. This difference was not statistically significant $(P=0.223)$ 
Table 4

Direct hospitalization cost comparison between ACE +7 group and CUS group after PSM.

\begin{tabular}{|c|c|c|c|c|}
\hline Costs (RMB), mean \pm SD & $\begin{array}{l}\text { ACE }+7 \text { group }(N= \\
50)\end{array}$ & $\begin{array}{l}\text { CUS group } \\
(\mathrm{N}=50)\end{array}$ & $\begin{array}{l}\text { Difference } \\
\text { (ACE }+7- \\
\text { CUS) }\end{array}$ & $\begin{array}{l}P \\
\text { value }\end{array}$ \\
\hline Total cost & $42675.0 \pm 5134.5$ & $\begin{array}{l}41426.3 \pm \\
7623.7\end{array}$ & 1248.8 & 0.349 \\
\hline Device cost & $23308.4 \pm 5702.0$ & $\begin{array}{l}19467.5 \pm \\
5946.7\end{array}$ & 3840.9 & 0.002 \\
\hline Treatment cost & $3287.0 \pm 3902.9$ & $\begin{array}{l}4412.4 \pm \\
4097.2\end{array}$ & -1125.4 & 0.176 \\
\hline Pharmaceutical cost & $6800.3 \pm 4169.0$ & $\begin{array}{l}7436.4 \pm \\
4481.4\end{array}$ & -636.0 & 0.478 \\
\hline Nursing cost & $555.6 \pm 275.8$ & $649.9 \pm 352.1$ & -94.3 & 0.149 \\
\hline Board and room cost & $723.0 \pm 636.0$ & $858.7 \pm 694.4$ & -135.7 & 0.325 \\
\hline Lab test cost & $2636.8 \pm 805.9$ & $\begin{array}{l}3132.3 \pm \\
1280.2\end{array}$ & -495.5 & 0.025 \\
\hline Examination cost & $3591.3 \pm 763.4$ & $\begin{array}{l}3792.1 \pm \\
1178.1\end{array}$ & -200.7 & 0.324 \\
\hline Transfusion cost & $20.0 \pm 141.4$ & $49.2 \pm 192.0$ & -29.2 & 0.400 \\
\hline Oxygen therapy cost & $44.3 \pm 8.4$ & $62.9 \pm 50.8$ & -18.7 & 0.012 \\
\hline TCM cost & $996.5 \pm 1034.8$ & $765.4 \pm 999.0$ & 231.1 & 0.275 \\
\hline Diagnosis cost & $376.0 \pm 88.7$ & $384.4 \pm 106.0$ & -8.4 & 0.677 \\
\hline Other cost & $335.8 \pm 355.5$ & $415.1 \pm 426.8$ & -79.3 & 0.328 \\
\hline $\begin{array}{l}\text { Total cost excluding intervention } \\
\text { cost }^{\star}\end{array}$ & $38357.8 \pm 5127.1$ & $\begin{array}{l}39983.1 \pm \\
7618.3\end{array}$ & -1625.2 & 0.223 \\
\hline \multicolumn{5}{|c|}{$\begin{array}{l}\text { *Intervention cost for } \mathrm{ACE}+7 \text { group is the service fee of integrated ultrasonic and electrosurgical } \\
\text { energy system ( } 800 \mathrm{RMB}) \text { and the cost of Harmonic ACE }+7 \text { shear ( } 3500 \mathrm{RMB}) \text { which has a separate } \\
\text { Billing-Code. Intervention cost for CUS group is service fee of ultrasonic shear system (1400 RMB) } \\
\text { which contains cost of ultrasonic shear and relevant service. }\end{array}$} \\
\hline
\end{tabular}

\section{Discussion}

While other studies show the device's clinical effectiveness, this is one of the first studies to use realworld evidence to demonstrate that the ACE +7 shear could improve intraoperative and postoperative outcomes without increasing the total hospitalization cost. This study's results are comparable to previous studies and shows that the ACE +7 shear with integrated energy system provides better hemostasis effectiveness than conventional ultrasonic shears. Previous studies have focused explicitly 
on the technical processes and modes of improvement associated with the ACE +7 , such as decreased thermal energy and increased burst energy, but have not investigated clinical outcomes related to the new ACE +7 technology compared to CUS. This study provides evidence that there are clinical benefits associated with using the ACE +7 in LPR procedures.

In particular, the new surgical shear was associated with decreased use of hemostatic clips and hemostatic drugs. A recent systematic review found that excessive bleeding was one of the most common LPR complications, affecting $12 \%$ of patients [12]. This is considerably higher than the $3 \%$ of patients who experience excessive bleeding in surgeries overall [13]. Excessive bleeding can cause hemodilution, hypothermia, consumption of clotting factors, and acidosis [14]. Greater bleeding control could lower surgical complications.

Similarly, in older populations, hemostasis medications are associated with a high risk of gastrointestinal bleeding [15]. Therefore, decreasing hemostatic drug usage is particularly important as older men are more likely to develop prostate cancer. Adopting medical innovations to reduce complications in the populations most affected by prostate cancer is essential to provide high-quality care and achieve better outcomes.

Additionally, the ACE +7 shear was associated with lower postoperative drainage duration and nearly a $50 \%$ reduction in drainage volume. Longer postoperative draining durations can increase the risk of infections [16]. These infections usually clear on their own but can cause urinary tract infections or more widespread infections throughout the body. In turn, using the ACE + 7 shear decreases the average draining duration for LPR patients, reducing the risk of irritating and potentially dangerous postoperative complications, and even antibiotic drug usage.

The ACE + 7 did not reduce total hospital costs for LPR patients but lowered several categorical costs. Most notably, patients in the ACE +7 group experienced cost savings through needing fewer hemostatic clips. While hemostatic clips are not extremely expensive, it is essential to cut costs wherever possible during surgery to compensate for the tool's higher price. The premium price of the ACE +7 shear is offset by cost reductions in treatment cost, pharmaceutical cost, nursing cost, and room and board cost. These cost savings are relevant results of the better clinical outcomes associated with the ACE +7 shears. For example, if patients in the ACE + 7 group needed fewer hemostatic drugs, they would have lower pharmaceutical costs.

Medical innovations such as the ACE + 7 shear with integrated energy system are essential to giving patients Value-Based Care (VBC) or care that offers people the best outcomes for the lowest costs. When evaluating the cost of a new technology, it is essential to take the total relevant costs into consideration, rather than narrowly focusing on the device price. For example, if a device improves a surgical process, perioperative hospitalization costs could be affected by the introduction of the new technology and should be analyzed comprehensively. In the context of VBC, the ACE + 7 technology results in better outcomes but does not increase overall costs. Although the tool itself costs the health system more money, there are areas for further cost savings that offset the device cost. 
VBC is essential if China wants to reach Universal Health Coverage. In 2009, China launched an extensive health reform plan to provide all citizens equal access to quality health and financial risk protection [17]. In response to current gaps in this plan, the WHO released recommendations to help China achieve the goal, such as improving quality of care through patient-centered care and VBC to overcome these shortfalls in the hopes of patients having better health, higher-quality care, and care at affordable costs [18]. Overall, increasing the ACE +7 shear uptake for LRPs can lead to high-quality, cost-effective care for patients with prostate cancer.

There are several limitations in this study. Firstly, in the initial study population, patients had more complex conditions in the ACE + 7 group than the CUS group. For example, the percentage of abnormal coagulation function and conducting lymph node dissection during surgery are both much higher in ACE + 7 group. Although we conducted PSM to control the difference, the inconsistence could not be entirely eliminated since some confounders could not be recorded in our study, such as the tumor size, TNM stage, prostate tissue condition, etc. There was also a loss of sample size due to the inconsistence of baseline characteristics. Secondly, we did not capture indirect costs, such as the patient care cost after discharge. Finally, Ruijin hospital is one of the top Tier hospital in China according to Hospital Rankings in China (Fudan 2020 version). The surgeons' surgical skill in Ruijin Hospital is relatively advanced compared to those in Tier hospital. As a result, the study results might not be the same in Tier hospitals. However, we assume the new technology with a stable clinical effectiveness could help to standardize the surgical procedures and reduce reliance on surgical skills, and so that could provide better performance for junior surgeon.

\section{Conclusions}

This real-world study demonstrated that the ACE +7 shear with integrated energy system improved clinical outcomes compared to the conventional ultrasonic shear for patients receiving LPRs. While the device cost more than a conventional ultrasonic shear, the ACE +7 can offer other cost savings for hospitals and health systems.

\section{List Of Abbreviations}

$\mathrm{ACE}+7$

Harmonic ACE + 7 Shear

CUS

Conventional ultrasonic shears

HIS

Electronic Hospital Information System

LPR

Laparoscopic prostate resections

LOS

Length of sray 
PSM

Propensity score matching

TCM

Traditional Chines medicine

VBC

Value-based care

\section{Declarations}

Ethics Approval and consent to participate: The study was reviewed and approved by Ethical Committee in September 2021 and all methods were performed in accordance with the relevant guidelines and regulations. Waiver of informed consent elements was approved by Ruijin Hospital Ethics Committee in Shanghai JiaoTong University School of Medicine as the research is a retrospective, observational study based on data from existing Electronical Hospital Information System and no human intervention was involved, and the research involves no more than minimal risk to subjects and will not adversely affect the rights and welfare of the subjects. Ethics committee name: Ruijin Hospital Ethics Committee, Shanghai JiaoTong University School of Medicine.

Ethics committee reference number: 2020-304

Consent for publication: Not applicable

Availability of data and materials: The datasets used and/or analysed during the current study are available from the corresponding author on reasonable request.

Competing interests: The authors declare that they have no competing interests

Funding: There are no funding resources to be reported or declared.

Author Contribution: Yi Gao and Danfeng Xu conceived and planned the experiments. Yi Gao, Yu Zhu, Fukang Sun verified the analytical methods. Yi Gao, Yu Zhu, Fukang Sun, Yuan Shao, Tao Huang, Wei He, Xin Xie, Lu Chen carried out the experiment. Debra Winberg wrote the manuscript with support from Yi Gao and Danfeng Xu. Danfeng Xu supervised the project. All authors provided critical feedback, discussed the results and contributed to the final manuscript.

Acknowledgements: Not applicable.

Author Information:

Affiliations:

Ruijin Hospital, Shanghai Jiao Tong University, School of Medicine, Shanghai, 200001, China

Yi Gao, Yu Zhu, Fukang Sun, Yuan Shao, Tao Huang, Wei He, Xin Xie, Lu Che, Danfeng Xu 
Tulane School of Public Health and Tropical Medicine, Department of Health Policy and Management, New Orleans, LA, USA

Debra Winberg

Corresponding Author: Danfeng Xu, MD. xdf12036@rjh.com.cn

\section{References}

1. Speight J, Holmes-Truscott E, Hendrieckx C, Skovlund S, Cooke D: Assessing the impact of diabetes on quality of life: what have the past 25 years taught us? Diabet Med 2020, 37(3):483-492.

2. Schatten H: Brief Overview of Prostate Cancer Statistics, Grading, Diagnosis and Treatment Strategies. Adv Exp Med Biol 2018, 1095:1-14.

3. Chaussy CG, Thüroff S: High-Intensity Focused Ultrasound for the Treatment of Prostate Cancer: A Review. J Endourol 2017, 31(S1):S30-S37.

4. Yu W, Zhou L: Early Diagnosis of Prostate Cancer from the Perspective of Chinese Physicians. J Cancer 2020, 11(11):3264-3273.

5. Chinese guidelines for diagnosis and treatment of prostate cancer 2018 (English version). Chin $\mathrm{J}$ Cancer Res 2019, 31(1):67-83.

6. Kondrup JD, Anderson F, Sylvester B, Branning M: The New HARMONIC ACE $®+7$ Shears: A Game Changer in Ultrasonic Hemostasis. Surg Technol Int 2014, 25:24-27.

7. Timm RW, Asher RM, Tellio KR, Welling AL, Clymer JW, Amaral JF: Sealing vessels up to $7 \mathrm{~mm}$ in diameter solely with ultrasonic technology. Med Devices (Auckl) 2014, 7:263-271.

8. Church JT, McLeod JS, Coughlin MA, Bergin IL, Perkins EM, Hoffman HR, Bilger M, Rojas-Peña A, Treadwell MC, Berman DR et al: An Early Investigation into Possible Alternatives to Stapled Hysterotomy in Open Fetal Surgery. Am J Perinatol 2019, 36(7):742-750.

9. Singleton D, Juncosa-Melvin N, Scoggins P, Paulin-Curlee G, Cummings J, Ricketts C: Intelligent Ultrasonic Energy: New Adaptive Tissue Technology in Harmonic Shears. Journal of Surgery 2020, 8(6):178-183.

10. Zurawin R, Pickron T, Blackstone R: Intelligent Ultrasonic Energy Delivered by HARMONIC® devices with Adaptive Tissue Techno. In.: Ethicon and Springer Healthcare; 2014.

11. Benedetto U, Head SJ, Angelini GD, Blackstone EH: Statistical primer: propensity score matching and its alternatives. Eur J Cardiothorac Surg 2018, 53(6):1112-1117.

12. Dokmak S, Ftériche FS, Aussilhou B, Lévy P, Ruszniewski P, Cros J, Vullierme MP, Khoy Ear L, Belghiti J, Sauvanet A: The Largest European Single-Center Experience: 300 Laparoscopic Pancreatic Resections. J Am Coll Surg 2017, 225(2):226-234.e222.

13. Curnow J, Pasalic L, Favaloro EJ: Why Do Patients Bleed? Surg J (N Y) 2016, 2(1):e29-e43.

14. Marietta M, Facchini L, Pedrazzi P, Busani S, Torelli G: Pathophysiology of bleeding in surgery. Transplant Proc 2006, 38(3):812-814. 
15. Franco Moreno Al, Martín Díaz RM, García Navarro MJ: Direct oral anticoagulants: An update. Med Clin (Barc) 2018, 151(5):198-206.

16. Tan H, Jin D, Sun J, Song J, Lu Y, Yin M, Chen X, Qu X, Liu C: Enlisting a Traditional Chinese Medicine to tune the gelation kinetics of a bioactive tissue adhesive for fast hemostasis or minimally invasive therapy. Bioact Mater 2021, 6(3):905-917.

17. Yip W, Fu H, Chen AT, Zhai T, Jian W, Xu R, Pan J, Hu M, Zhou Z, Chen Q et al: 10 years of health-care reform in China: progress and gaps in Universal Health Coverage. Lancet 2019, 394(10204):11921204.

18. Zhang J, Xian TZ, Wu MX, Li C, Pan Q, Guo LX: Comparison of the effects of twice-daily exenatide and insulin on carotid intima-media thickness in type 2 diabetes mellitus patients: a 52-week randomized, open-label, controlled trial. Cardiovasc Diabetol 2020, 19(1):48.

\section{Figures}

\section{2 cases meet inclusion and exclusion criteria}

\section{3 included for description analysis of baseline $(\mathrm{ACE}+7: \mathrm{ACE}+=70: 123)$}

\section{1: 1 propensity score matching}

100 included for endpoints comparison $(\mathrm{ACE}+7: \mathrm{ACE}+=50: 50)$ 
Figure 1

Patient Population Selection

Page 15/15 